\title{
Self-affine fractal electrodeposited gold surfaces: Characterization by scanning tunneling microscopy
}

\author{
L. Vázquez, ${ }^{1}$ R. C. Salvarezza, ${ }^{3}$ P. Ocón, ${ }^{2}$ P. Herrasti, ${ }^{2}$ J. M. Vara, ${ }^{2}$ and A. J. Arvia ${ }^{3}$ \\ ${ }^{1}$ Instituto de Ciencia de Materiales, Consejo Superior de Investigaciones Científicas, Departamento de Física Aplicada, C-XII, \\ Universidad Autónoma de Madrid 28049, Madrid, Spain \\ ${ }^{2}$ Departamento de Química Física Aplicada C-II, Universidad Autónoma de Madrid, 28049 Madrid, Spain \\ ${ }^{3}$ Instituto de Investigaciones Fisicoquímicas Teóricas y Aplicadas, \\ Sucursal 4, Casilla de Correo 16, (1900) La Plata, Argentina
}

(Received 14 July 1993)

\begin{abstract}
The morphological evolution of columnar gold electrodeposits grown at $100 \mathrm{~nm} \mathrm{~s}^{-1}$ by electroreducing a gold oxide layer on a gold cathode has been studied at a nanometer level by scanning tunneling microscopy. The interface thickness $(\xi)$ depends on the scan length $(L)$ as $\xi \propto L^{\alpha}$ with $\alpha=0.49 \pm 0.07$ for $L>d_{s}$, where $d_{s}$ is the average top columnar size, and $\alpha=0.90 \pm 0.07$ for $L<d_{s}$. These results prove that the growing surface can be described as a self-affine fractal for length scales greater than the columnar size. Conversely, the columnar surface approaches the behavior of an Euclidean surface.
\end{abstract}

PACS number(s): 81.15.Lm, 68.55.Jk, 05.40. $+\mathrm{j}$, 05.70.Ln

\section{INTRODUCTION}

A relatively large number of physical and chemical processes are related to the development of complex structures involving rough surfaces. This is the case of metal electrodeposition from either aqueous solutions or hydrous metal oxide layers on a conducting substrate [1]. Depending on the experimental conditions, metal electrodeposits can exhibit diffusion-limited aggregation, or dense radial or columnar patterns. Columnar structures develop at intermediate growth rates in the $15<v<150$ $\mathrm{nm} \mathrm{s}^{-1}$ range [2]. The interface of columnar deposits has been described as either self-similar [3] or self-affine fractals [4]. As concluded from large-scale computer simulations, the Eden model generates compact structures with self-affine fractal surfaces [5], whereas continuum models predict compact structures with self-similar surfaces [3].

Several attempts to characterize the surfaces of metal electrodeposits as fractals have been reported $[1,6,7]$. Recently, it has been shown that when the scale length $(L)$ is smaller than the average top columnar size $\left(d_{s}\right)$, the interface of two-dimensional (2D) columnar copper electrodeposits behaves as a self-affine fractal, fulfilling the laws $\xi \propto L^{\alpha}$ with $\alpha \cong \frac{1}{2}$, and $\xi \propto \bar{h}^{\beta}$ with $\beta>1$, where $\xi$ is the root-mean-square roughness, and $\bar{h}$ is the average column height. In this particular case $d_{s}$ has been estimated as $500 \mu \mathrm{m}$ [8]. The value $\alpha=\frac{1}{2}$ is consistent with that expected from the Eden model [5] used for describing the evolution and properties of different types of aggregates, but this is not the case for the value of $\beta$. According to the Eden model the value $\beta=\frac{1}{3}$ should be expected instead of $\beta>1$ [5]. Nevertheless, as the preceding analysis [8] was restricted to scale length values in the $5-500-\mu \mathrm{m}$ range, a large extrapolation was required to compare the results to computer simulation data. This inconvenience can be overcome when high-resolution data become available. These data would offer the possibility of checking the validity of models. For this purpose scanning tunnel- ing microscopy (STM) would be an adequate technique because of its high $3 \mathrm{D}$ resolution and nondestructive character [9]. In fact, STM has been used recently to analyze the fractal roughness of vapor-deposited gold films $[10,11]$. This type of STM fractal study can be extended to a number of electrodeposited metal films.

In this work the STM fractal analysis of columnar gold films covering a relatively broad range of film thicknesses is reported. Dynamic scaling analysis of interface growth [12] from STM data has allowed us to prove the selfaffine character of electrodeposited gold film surfaces, and to evaluate the exponent $\alpha$ from the $\log _{10} \xi$ vs $\log _{10} L$ relationships.

\section{EXPERIMENT}

Gold films on gold cathodes were grown at $100 \mathrm{~nm} \mathrm{~s}^{-1}$ by electroreducing a hydrous gold oxide layer previously formed at $2.5 \mathrm{~V}$ (vs the reversible hydrogen electrode) in $0.5 \mathrm{M}$ aqueous sulfuric acid using a conventional electrochemical setup at $298 \mathrm{~K}$. Details about gold film preparation are given elsewhere [13]. These films exhibit a rough surface and a porous mass with a uniform apparent density which is close to that of bulk gold oxide [13]. The average gold film thickness $\delta$ becomes proportional to $q$, the electrodeposited charge density defined as the electrodeposited charge per unit of geometric substrate area by the relationship [14]

$$
\delta=M q / z F \rho,
$$

where $M$ and $\rho$ are the molecular weight and the density of gold oxide, and $z F$ is the corresponding molar charge. From Eq. (1), and taking $M=440 \mathrm{~g} \mathrm{~mol}^{-1}, \rho=11$ $\mathrm{g} \mathrm{cm}^{-3}$, and $z=6$ for gold oxide, $\delta$ can be estimated from $q$ values [14]. Values of $\delta$ in the 100-12000-nm range were obtained by adjusting the amount (thickness) of hydrous gold oxide previously produced.

The surface morphology of gold films was studied in air using two different STM microscopes, namely a Nano- 
scope II STM and a homemade piezotube STM controlled through ATOMIS electronics. Both STM's were calibrated by imaging highly oriented pyrolytic graphite (HOPG) surfaces. To minimize the influence of artifacts due to tip geometry, different platinum tips were used. These tips were made from 0.5 -mm-diam platinum wires directly by cutting. STM measurements were performed using $0.05-\mathrm{V}$ bias voltage with the tip positive at $1-2-\mathrm{nA}$ constant current. Data were acquired in a fully automated work station, and stored as digitized images with $400 \times 400$ pixels.

\section{RESULTS AND DISCUSSION}

STM images of electrodeposited gold film surfaces covering the $100<\delta<12000-\mathrm{nm}$ range are shown in Figs.
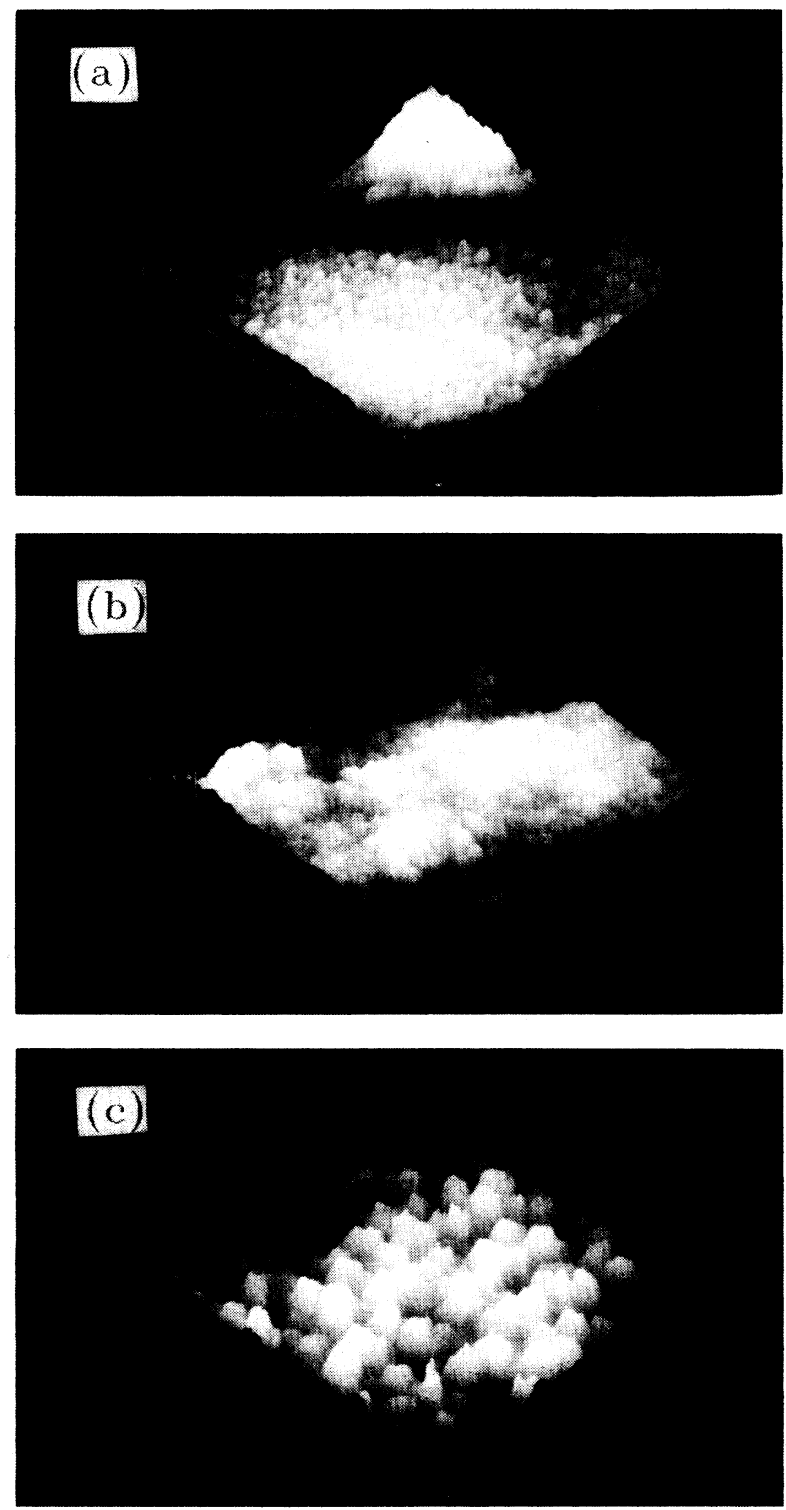

FIG. 1. 3D STM images of gold electrodeposits. (a) $650 \times 650 \mathrm{~nm}^{2}, \delta=160 \mathrm{~nm}$. (b) $700 \times 700 \mathrm{~nm}^{2}, \delta=560 \mathrm{~nm}$. (c) $700 \times 700 \mathrm{~nm}^{2}, \delta=9100 \mathrm{~nm}$. Note that the $z$ scale is the same in the three images. 1(a)-1(c). These images reveal a deposit formed by a rounded top columnar structure. As $\delta$ increases, the surface becomes rougher due to the preferential growth of some columns as compared to other ones, this effect being caused by shadowing. In this case, $d_{s}$ increases with $\delta$. The value of $\xi_{\mathrm{STM}}$, the root-mean-square roughness measured from STM images, increases initially with both $\delta$ and $S$, the total scan length (Fig. 2), but for $\delta=160 \mathrm{~nm}$ and $S>1.5 \mu \mathrm{m}$ a constant value of $\xi_{\mathrm{STM}}$ is reached.

Furthermore, for a given $\delta$ value, the deposit surface imaged at different magnifications looks very similar, as expected for a fractal surface [Figs. 3(a) -3(b)].

To characterize the fluctuating interfaces of gold electrodeposits, dynamic scaling analysis of surface growth has been applied $[5,12]$. Accordingly, $\xi^{i}(L)$, the surface width in the $i$ direction, for a sample of lateral dimension $L$, can be defined as the root-mean-square roughness given by

$$
\xi^{i}(L)=\left[(1 / L) \sum_{j}\left[h_{j}-h_{0}\right]^{2}\right]^{1 / 2},
$$

where $h_{j}$ is the deposit height measured along the $i$ direction at the $j$ position, and $h_{0}$ is the average height of the sample. In addition, $\xi$ and $L$ are related through the equation

$$
\xi^{i}(L, h) \propto L^{\alpha} f(x),
$$

where $f(x)=h_{0} / L^{\gamma}$. The function $f(x)$ has the following properties: $f(x)=$ const for $x \Longrightarrow \infty$, and $f(x)=x^{\alpha / \gamma}$ for $x \Longrightarrow 0$. After a certain critical thickness (growth time), $\xi^{i}$ reaches a steady state, and Eq. (3) becomes

$$
\xi^{i}(L) \propto L^{\alpha} .
$$

These dynamic scaling concepts can be applied to STM data derived from electrodeposited gold surfaces as

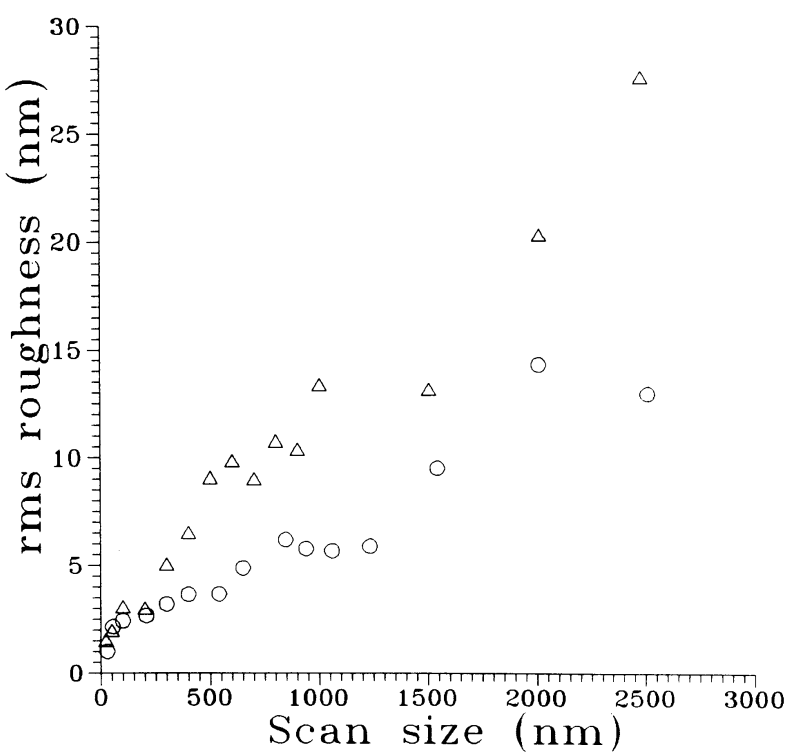

FIG. 2. Dependence of $\xi_{\mathrm{STM}}$, the rms roughness of the STM image, on $S$, the total scan length, for $\delta=9100(\Delta)$ and $\delta=160$ $\mathrm{nm}(0)$. 
(a)

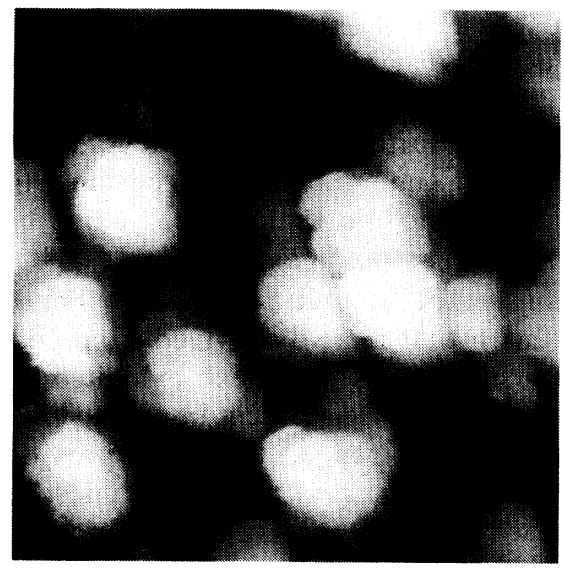

(b)

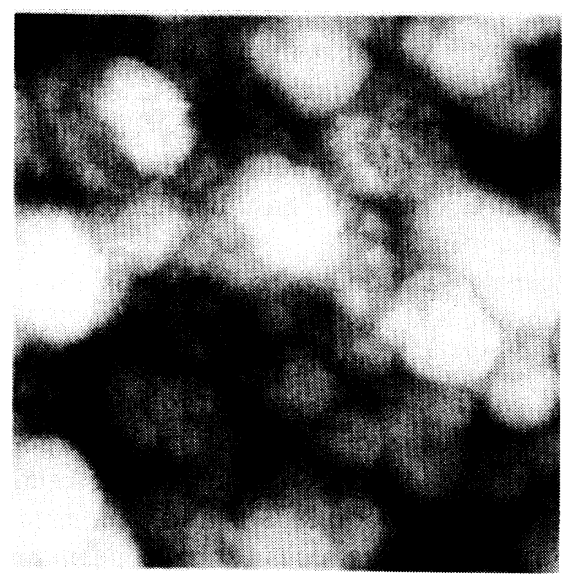

FIG. 3. STM top view images at different magnifications of a gold film with $\delta=9100 \mathrm{~nm}$. (a) $300 \times 300 \mathrm{~nm}^{2}$; (b) $1280 \times 1280$ $\mathrm{nm}^{2}$.

$\xi^{i}=\xi_{\mathrm{STM}}^{i}$, where $\xi_{\mathrm{STM}}^{i}$ is the root-mean-square roughness determined from the STM scans in the $i$ direction $(i=x$ and $y$ ). Accordingly, we have evaluated the value of $\xi_{\text {STM }}^{i}$ over each STM scan segment of length $L_{s}$ along the $x$ and $y$ directions after the standard plane correction. More explicitly, we have used

$$
\xi_{\mathrm{STM}}^{i}\left(L_{s}\right) \propto\left[\left(1 / L_{s}\right) \sum_{j}\left[h_{j}-\bar{h}_{s}\right]^{2}\right]^{1 / 2}
$$

and

$$
\xi_{\mathrm{STM}}^{i} \propto L_{s}^{\alpha},
$$

where $\bar{h}_{s}$ is the average height of the surface profile of length $L_{s}$ measured by STM in either the $x$ or $y$ directions. For each scan 360 data pairs $\left(L_{s}, \xi_{\mathrm{STM}}^{i}\right)$ have been obtained, $L_{s}$ being varied from $S / 64$ to almost $S$. Finally, for each $L_{s}$ the corresponding $\xi_{\mathrm{STM}}^{i}$ value represents the average of 400 scans of the same image. However, as already reported $[10,15], \log _{10} \xi_{\text {STM }}^{i}$ vs $\log _{10} L_{s}$ plots obtained in the $y$ direction sometimes presented anomalous $\alpha$ values due to either drift effects or noise arising from low-frequency mechanical vibrations. For this reason, the dynamic scaling analysis was restricted to those STM scans made in the fast $x$ direction, and presented as $\log _{10} \xi_{\text {STM }}^{x}$ vs $\log _{10} L_{s}$ plots for each STM image.

It should be noted that although for computersimulated fractals data covering four to five orders of magnitude in scale length are usually required for logarithmic fittings [1], for experimental systems the situation is less ambitious, as frequently the fractal behavior is restricted to a certain scale length. Accordingly, fractal structures of real objects are characterized by log-log plots covering scale lengths of at least one order of magnitude or thereabouts [16].

Typical plots obtained for electrodeposited gold films with $\delta=160 \mathrm{~nm}$ for three different STM images, and $\delta=12000 \mathrm{~nm}$ for four different STM images, are shown in Fig. 4. In the former case (Fig. 4, plot $A$ ) two linear regions with slopes $\alpha_{1 A}=0.83 \pm 0.06$ and $\alpha_{2 A}=0.54$ \pm 0.06 , respectively, and a crossing point at $\log _{10} L_{0}=2.5$, can be observed. Similarly, in the latter case (Fig. 4, plot $B$ ) slopes $\alpha_{1 B}=0.79 \pm 0.06$ and $\alpha_{2 B}=0.41 \pm 0.06$ are obtained for $\log _{10} L_{s}$ values smaller and greater than $L_{0}=2.9$, respectively. In both cases the value of $L_{0}$ was on the order of $d_{s}$. However, as already reported [17] the dynamic scaling method applied to STM images with $256 \times 256$ size underestimates the value of $\alpha$ for $\alpha>0.6$. Thus in our case $\alpha_{1}$ must be corrected to obtain $\bar{\alpha}_{1}$, the true value of $\alpha_{1}$, in contrast to the value of $\alpha_{2}$, which coincides with $\bar{\alpha}_{2}$, the true value of $\alpha_{2}$.

Average values of $\bar{\alpha}_{1}, \bar{\alpha}_{2}$, and $L_{0}$, as well as $N_{1}$ and $N_{2}$, the number of averaged STM images for each sample, are summarized in Table I. Data demonstrate that the value of $\bar{\alpha}$ becomes independent of $\delta$, at least within the experimental errors. These results, which were taken using different tips, have allowed us to discard some appreciable effects of the tip on the fractal analysis of STM images. The average $\bar{\alpha}$ values are $\left\langle\bar{\alpha}_{1}\right\rangle=0.90 \pm 0.07$ $\left(N_{1}=153\right)$ and $\left\langle\bar{\alpha}_{2}\right\rangle=0.49 \pm 0.07\left(N_{2}=128\right)$.

Thus for $L_{s}<d_{s},\left\langle\bar{\alpha}_{1}\right\rangle \cong 1$. This fact suggests that a Euclidean behavior of the surface is attained as the columnar surface becomes smoother. Otherwise, for

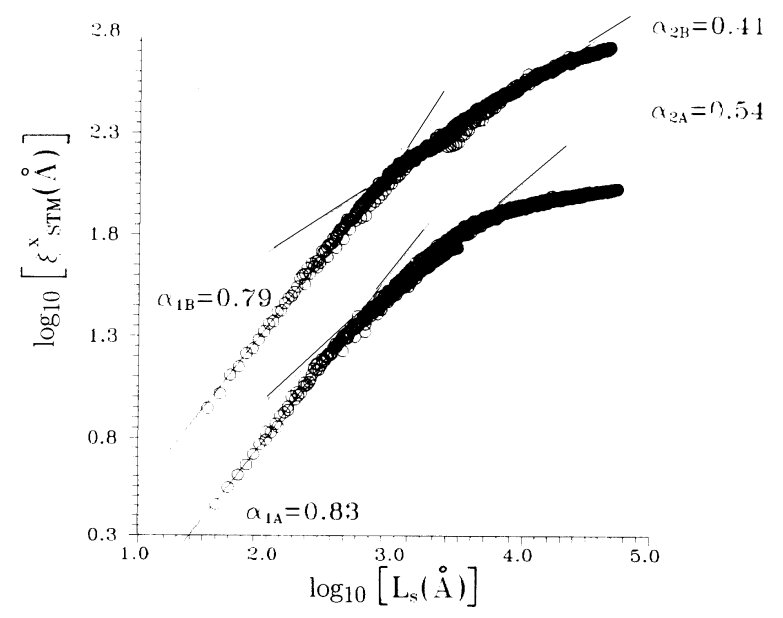

FIG. 4. $\log _{10} \xi_{\mathrm{STM}}^{x}$ vs $\log _{10} L_{s}$ plots calculated for $\delta=160$ (plot $A)$ and $9100 \mathrm{~nm}$ (plot $B$ ). Values of $\alpha$ below $\left(\alpha_{1}\right)$ and above $\left(\alpha_{2}\right)$ the crossing point $\left(L_{0}\right)$ are given. 
TABLE I. Values of $\bar{\alpha}_{1}, \bar{\alpha}_{2}$, and $L_{0}$ resulting from gold films of different values of $\delta . N_{1}$ and $N_{2}$ denote the number of STM images used to derive $\bar{\alpha}_{1}$ and $\bar{\alpha}_{2}$ values, respectively.

\begin{tabular}{rcccrc}
\hline \hline$\delta(\mathrm{nm})$ & $\bar{\alpha}_{1}$ & $N_{1}$ & $\bar{\alpha}_{2}$ & $N_{2}$ & $L_{0}(\mathrm{~nm})$ \\
\hline $50^{\mathrm{a}}$ & $0.96 \pm 0.07$ & 33 & $0.43 \pm 0.09$ & 17 & 24 \\
160 & $0.89 \pm 0.09$ & 23 & $0.41 \pm 0.05$ & 9 & 25 \\
500 & $0.91 \pm 0.06$ & 29 & $0.50 \pm 0.06$ & 32 & 30 \\
560 & $0.89 \pm 0.04$ & 17 & $0.50 \pm 0.06$ & 18 & 32 \\
2800 & $0.93 \pm 0.07$ & 23 & $0.52 \pm 0.07$ & 16 & 49 \\
9100 & $0.90 \pm 0.06$ & 28 & $0.51 \pm 0.08$ & 24 & 50 \\
11500 & $0.86 \pm 0.06$ & 21 & $0.45 \pm 0.06$ & 12 & 79 \\
& & & & \\
$\left\langle\bar{\alpha}_{1}\right\rangle=0.90 \pm 0.07$ & 174 & $\left\langle\bar{\alpha}_{2}\right\rangle=0.49 \pm 0.07$ & 128 & \\
\hline \hline
\end{tabular}

${ }^{\text {a }}$ This sample was prepared by electroreducing a $50-\mathrm{nm}$ gold oxide layer formed on $850-\mathrm{nm}$-thick vapor-deposited gold film by electro-oxidation in $0.5 \mathrm{M} \mathrm{H}_{2} \mathrm{SO}_{4}$ at $2 \mathrm{~V}$ with respect to the reversible hydrogen electrode.

$L_{s}>d_{s}$ a rough self-affine fractal surface is approached (see Fig. 5).

It has been reported that gold electrodeposits immersed in aqueous solution exhibit a roughness relaxation that fits the kinetics of particle coalescence controlled by surface diffusion $[13,18]$, i.e.,

$$
\left(d_{s} / 2\right)^{4}=2 \gamma a^{4} D t / k T,
$$

where $\gamma$ is the gold surface tension, $a$ is the lattice parameter, $k$ is Boltzmann's constant, and $D$ is the surface diffusion coefficient of deposited particles. Taking $a=4 \times 10^{-8} \mathrm{~cm}, \gamma=1000$ erg cm ${ }^{-1}, D=10^{-14} \mathrm{~cm}^{2} \mathrm{~s}^{-1}$ $[13,19]$, and $T=298 \mathrm{~K}$ for $t=100 \mathrm{~s}$, it results that $d_{s} \cong 20 \mathrm{~nm}$, a figure which is close to the columnar top

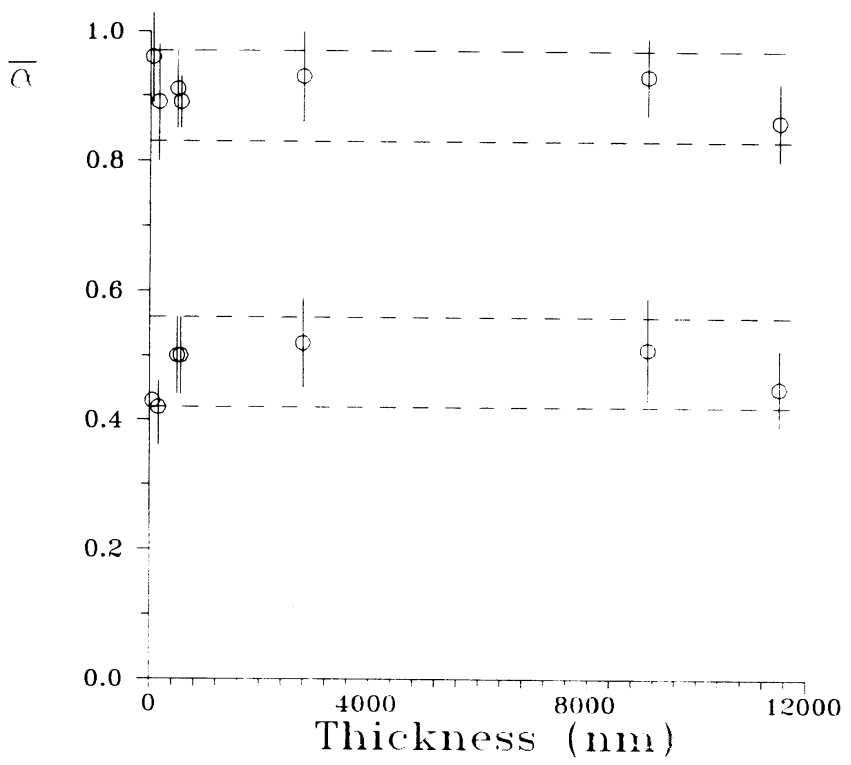

FIG. 5. The $\bar{\alpha}_{1}$ vs $\delta$ (top values) and $\bar{\alpha}_{2}$ vs $\delta$ (bottom values) plots. Error bars are indicated. For each plot the dashed lines correspond to the error of the final averaged value. size of these gold films as imaged by STM. Accordingly, irregularities at the deposit surface which are smaller than the diffusion length, i.e., of sizes close to $d_{s}$, are leveled off by surface diffusion of gold atoms during the preparation time interval. According to these results, one must conclude that the self-affine character of the columnar structure $\left(\left\langle\bar{\alpha}_{2}\right\rangle \cong \frac{1}{2}\right.$ for $\left.\left.L_{s}\right\rangle d_{s}\right)$ must be due to column height fluctuations (Fig. 1). This conclusion contrasts with that recently derived from columnar $\mathrm{Cu}$ electrodeposit surfaces [8], which exhibited a self-affine character only for those surfaces involving sizes of columns in the micrometer range. Our data provide evidence that those columns imaged by optical microscopy [8] can be described as a collection of small nanometer-size columns which can be resolved only through STM imaging. This is clearly seen in Fig. 6, where nanometer-size columns agglomerate yielding micrometer-size structures. Thus the irregularities which give self-affine character to the surface are actually caused by height fluctuations of small columns, as earlier concluded from vapor-deposited gold films $[10,17]$, rather than by fluctuations of their own columnar surfaces.

Recently, a value of $\alpha$ which is consistent with the Eden model [5] has been reported for 2D copper electrodeposits [8]. However, the value $\left\langle\bar{\alpha}_{2}\right\rangle \cong \frac{1}{2}$ for $L_{s}>d_{s}$ obtained in this work for 3D gold electrodeposits differs from the value of $\alpha$ predicted for $3 D$ large-scale computer simulations of the Eden model [5]. Similar discrepancies between experimental data and theoretical expectations for other growth processes have been previously found [11]. Thus further work is still necessary for a comprehensive understanding of the meaning of the $\left\langle\bar{\alpha}_{2}\right\rangle$ value.

Finally, $\mathcal{D}$, the local fractal dimension of the deposited gold surface, can be obtained from

$$
\mathcal{D}=3-\alpha \text {. }
$$

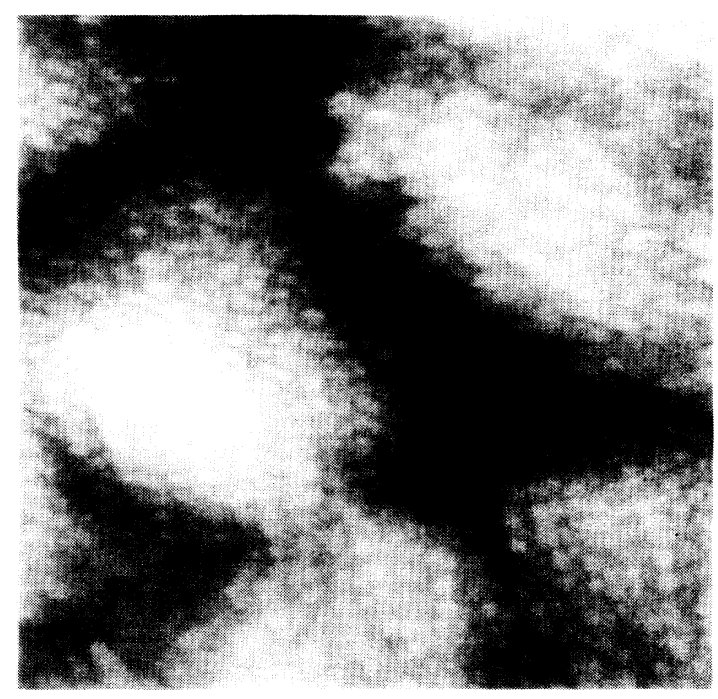

FIG. 6. Top view STM image $\left(1230 \times 1230 \mathrm{~nm}^{2}\right)$ of a gold film with $\delta=160 \mathrm{~nm}$. Note that micrometer-size structures are formed by a large number of nanometer-size elements. 
Thus it results that $D_{1}=2$ and $D_{2}=2.5$ for the two regimes, respectively. The value of $\mathscr{D}_{2}$ agrees with values earlier reported for gold deposits using the perimeterarea method applied to STM images [20], and by study- ing diffusion-controlled electrochemical reactions in this type of gold surface [21]. This confirms the capability of STM to characterize the dynamics of rough conducting surfaces at high-resolution levels.
[1] P. Meakin, Crit. Rev. Solid State Mater. Sci. 13, 147 (1987).

[2] B. Movchan and A. V. Demchishin, Fiz. Met. Metalloved. 28, 653 (1969) [Phys. Met. Metallogr. (USSR) 28, 83 (1969)].

[3] R. P. U. Karunasiri, R. Bruinsmà, and J. Rudnick, Phys. Rev. Lett. 62, 788 (1989).

[4] P. Meakin, P. Ramanlal, L. M. Sander, and R. C. Ball, Phys. Rev. A 34, 509 (1986); J. Krug and P. Meakin, Phys. Rev. A 43, 900 (1991).

[5] T. Vicsek, Fractal Growth Phenomena (World Scientific, Singapore, 1989).

[6] F. Sagues, F. Mas, M. Villarrasa, and J. Costa, J. Electroanal. Chem. 278, 351 (1990), and references therein.

[7] R. de Levie, J. Electroanal. Chem. 281, 1 (1990).

[8] G. L. M. K. S. Kahanda, X. Zou, R. Farrell, and P. Wong, Phys. Rev. Lett. 68, 3741 (1992).

[9] G. Binning, H. Rohrer, Ch. Gerber, and E. Weibel, Phys. Rev. Lett. 49, 57 (1982).

[10] R. C. Salvarezza, L. Vázquez, P. Herrasti, P. Ocón, J. M. Vara, and A. J. Arvia, Europhys. Lett. 20, 727 (1992).

[11] J. Krim, I. Heyvaert, C. Van Haesendonck, and Y. Bruynseraede, Phys. Rev. Lett. 70, 57 (1993).
[12] F. Family, Physica A 168, 561 (1990).

[13] C. Alonso, R. C. Salvarezza, J. M. Vara, A. J. Arvia, L. Vázquez, A. Bartolomé, and A. M. Baró, J. Electrochem. Soc. 137, 2161 (1990).

[14] L. Vázquez, A. Bartolomé, A. M. Baró, C. Alonso, R. C. Salvarezza, and A. J. Arvia, Surf. Sci. 215, 171 (1989).

[15] M. W. Mitchell and D. A. Bonnel, J. Mater. Res. 5, 2244 (1990).

[16] B. H. Kaye, in The Fractal Approach to Hetereogeneous Chemistry, edited by D. Avnir (Wiley, New York, 1990).

[17] L. Vázquez, R. C. Salvarezza, P. Herrasti, P. Ocón, J. M. Vara, and A. J. Arvia, Appl. Surf. Sci. 70/71, 413 (1993).

[18] M. Zinke-Allmang and L. C. Feldman, Appl. Surf. Sci. 52, 357 (1991).

[19] P. García, M. Gómez, R. C. Salvarezza, and A. J. Arvia, J. Electroanal. Chem. (to be published).

[20] J. M. Gómez Rodríguez, L. Vázquez, A. M. Baró, C. Alonso, R. C. Salvarezza, J. M. Vara, and A. J. Arvia, J. Phys. Chem. 96, 347 (1992).

[21] P. Ocón, P. Herrasti, L. Vázquez, R. C. Salvarezza, J. M. Vara, and A. J. Arvia, J. Electroanal. Chem. 319, 101 (1991). 

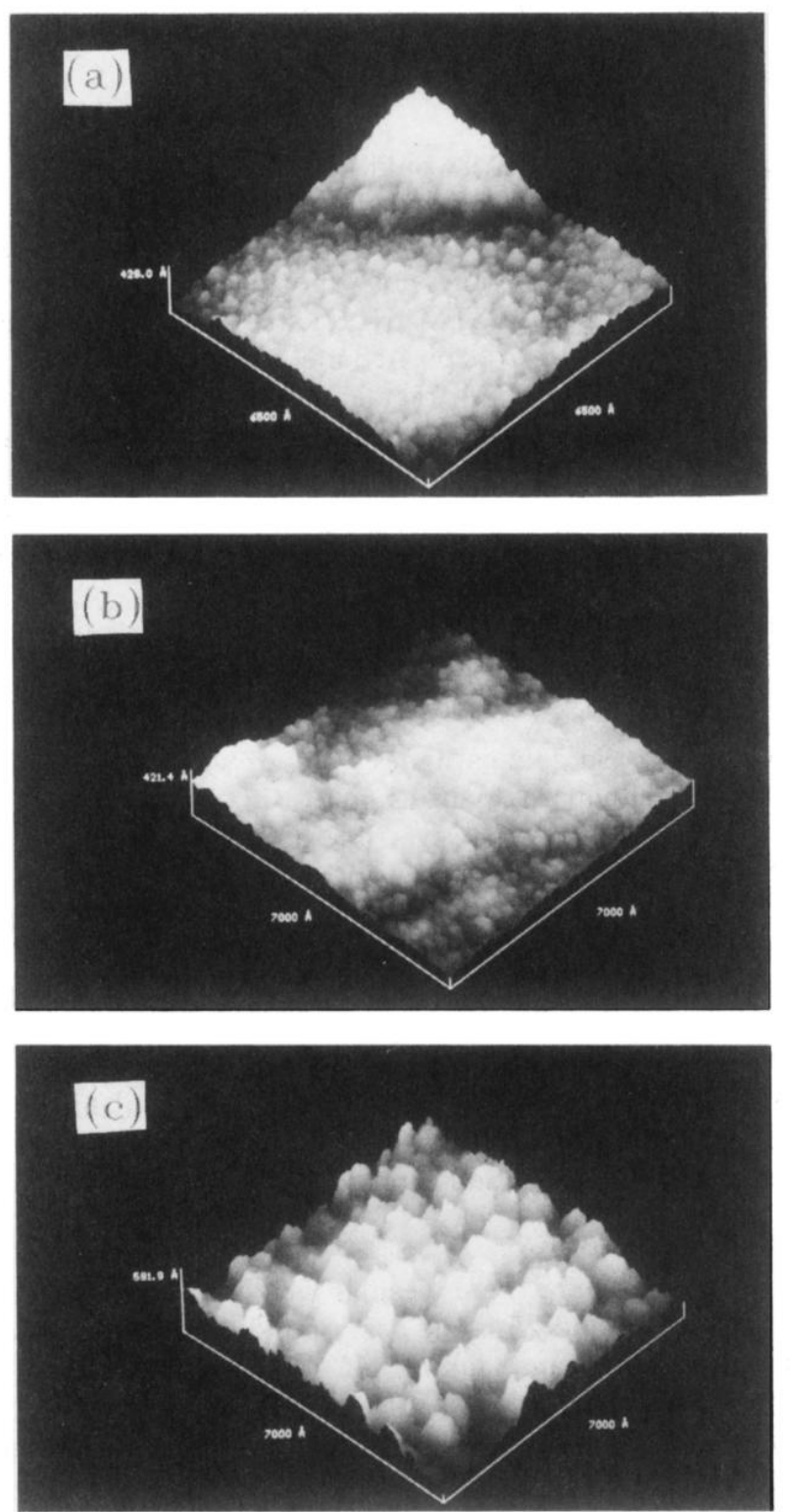

FIG. 1. 3D STM images of gold electrodeposits. (a) $650 \times 650 \mathrm{~nm}^{2}, \delta=160 \mathrm{~nm}$. (b) $700 \times 700 \mathrm{~nm}^{2}, \delta=560 \mathrm{~nm}$. (c) $700 \times 700 \mathrm{~nm}^{2}, \delta=9100 \mathrm{~nm}$. Note that the $z$ scale is the same in the three images. 
(a)

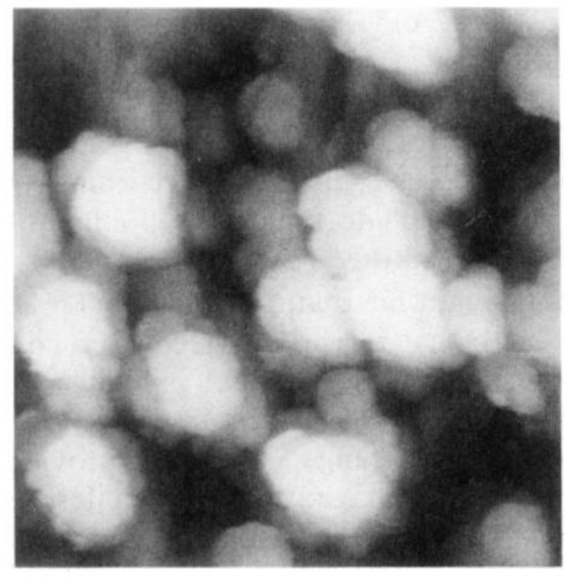

(b)

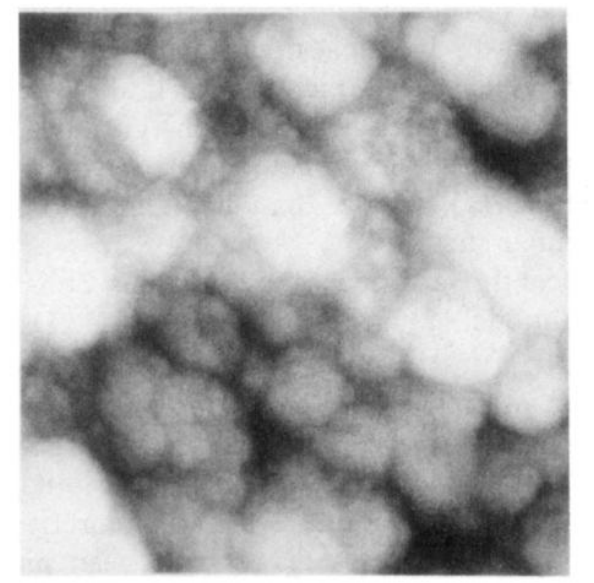

FIG. 3. STM top view images at different magnifications of a gold film with $\delta=9100 \mathrm{~nm}$. (a) $300 \times 300 \mathrm{~nm}^{2}$; (b) $1280 \times 1280$ $\mathrm{nm}^{2}$. 


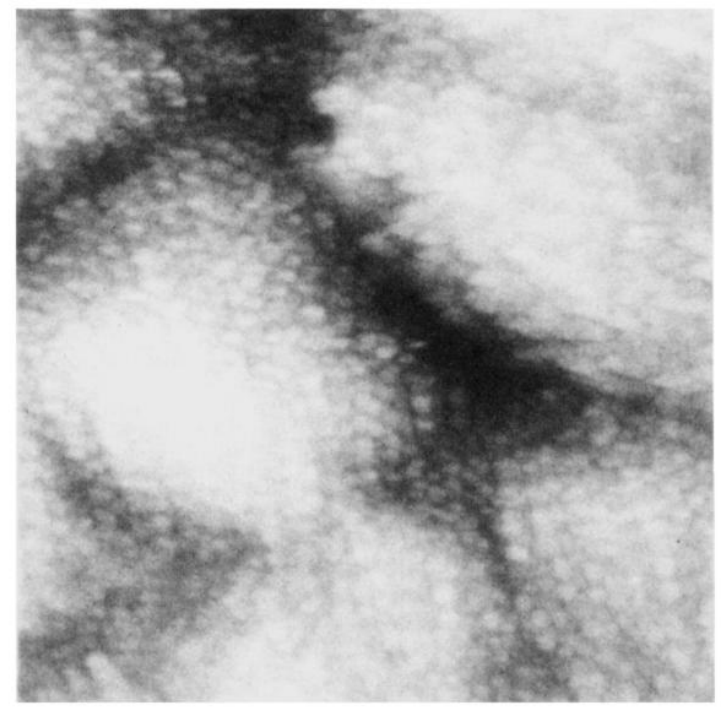

FIG. 6. Top view STM image $\left(1230 \times 1230 \mathrm{~nm}^{2}\right)$ of a gold film with $\delta=160 \mathrm{~nm}$. Note that micrometer-size structures are formed by a large number of nanometer-size elements. 